OPEN ACCESS

Edited by:

Yong Zhao,

Institute of Zoology

(CAS), China

Reviewed by:

Giovanna Lombardi,

King's College London,

United Kingdom

Femke Broere,

Utrecht University, Netherlands

*Correspondence:

Jin Lin

linjinzju@zju.edu.cn;

Song Guo Zheng

szheng1@hmc.psu.edu

Specialty section:

This article was submitted to Immunological Tolerance and Regulation,

a section of the journal

Frontiers in Immunology

Received: 11 March 2018 Accepted: 04 July 2018

Published: 18 July 2018

Citation:

Chen W, Wang J, Xu Z, Huang F, Qian W, Ma J, Wee Hb, Lewis GS,

June RR, Schafer PH, Lin J and

Zheng SG (2018) Apremilast Ameliorates Experimental Arthritis via

Suppression of Th1 and Th17 Cells and Enhancement of $\mathrm{CD}^{+}{ }^{+} \mathrm{Foxp} 3^{+}$ Regulatory T Cells Differentiation.

Front. Immunol. 9:1662. doi: 10.3389/fimmu.2018.01662

\section{Apremilast Ameliorates Experimental Arthritis via Suppression of Th1 and Th17 Cells and Enhancement of CD4 ${ }^{+}$Foxp3 ${ }^{+}$ Regulatory $\mathrm{T}$ Cells Differentiation}

\author{
Weiqian Chen ${ }^{1,2}$, Julie Wang ${ }^{2}$, Zhenjian $\mathrm{Xu}^{2,3}$, Feng Huang ${ }^{3}$, Wenbin Qian ${ }^{4}$, Jilin $\mathrm{Ma}^{5}$, \\ Hwa bok Wee ${ }^{6}$, Gregory S. Lewis ${ }^{6}$, Rayford R. June ${ }^{2}$, Peter H. Schafer ${ }^{7}$, Jin Lin ${ }^{1 *}$ \\ and Song Guo Zheng ${ }^{2 *}$
}

\begin{abstract}
'Division of Rheumatology, First Affiliated Hospital, College of Medicine, Zhejiang University, Hangzhou, China, ${ }^{2}$ Division of Rheumatology, Department of Medicine, Penn State University Hershey College of Medicine, Hershey, PA, United States, ${ }^{3}$ Department of Clinical Immunology, Third Affiliated Hospital, Sun Yat-sen Memorial Hospital of Sun Yat-sen University, Guangzhou, China, ${ }^{4}$ Division of Hematology, First Affiliated Hospital, College of Medicine, Zhejiang University, Hangzhou, China, ${ }^{5}$ Division of Rheumatology, Immunology, and Nephrology, Zhejiang Traditional Chinese Medicine and Western Medicine Hospital, Hangzhou, China, ${ }^{6}$ Department of Orthopaedics and Rehabilitation, Penn State University Hershey College of Medicine, Hershey, PA, United States, ${ }^{7}$ Translational Development, Inflammation and Immunology, Celgene Corporation, Summit, NJ, United States
\end{abstract}

Apremilast is a novel phosphodiesterase 4 (PDE4) inhibitor suppressing immune and inflammatory responses. We assessed the anti-inflammatory effects of Apremilast in type II collagen (CII)-induced arthritis (CIA) mouse model. To determine whether Apremilast can ameliorate arthritis onset in this model, Apremilast was given orally at day 14 after Cll immunization. Bone erosion was measured by histological and micro-computed tomographic analysis. Anti-mouse Cll antibody levels were measured by enzyme-linked immunosorbent assay, and Th17, Th1 cells, and CD4+Foxp3+ regulatory T (Treg) cells were assessed by flow cytometry in the lymph nodes. Human cartilage and rheumatoid arthritis (RA) synovial fibroblasts (RASFs) implantation in the severe combined immunodeficiency mouse model of RA were used to study the role of Apremilast in the suppression of RASF-mediated cartilage destruction in vivo. Compared with untreated and vehicle control groups, we found that Apremilast therapy delayed arthritis onset and reduced arthritis scores in the CIA model. Total serum IgG, IgG1, IgG2a, and IgG2b were all decreased in the Apremilast treatment groups. Moreover, Apremilast markedly prevented the development of bone erosions in CIA mice by CT analysis. Furthermore, in the Apremilast treated group, the frequency of Th17 cells and Th1 cells was significantly decreased while Treg cells' frequency was significantly increased. The high dose of Apremilast ( $25 \mathrm{mg} / \mathrm{kg}$ ) was superior to low dose $(5 \mathrm{mg} / \mathrm{kg})$ in treating CIA. Apremilast treatment reduced the migratory ability of RASFs and their destructive effect on cartilage. Compared with the model group, Apremilast treatment significantly reduced the RASFs invasion cartilage scores in both primary implant and contralateral implant models. Our data suggest that Apremilast is effective in treating autoimmune arthritis and preventing the bone erosion in the CIA model, implicating its therapeutic potential in patients with RA.

Keywords: rheumatoid arthritis, collagen Cll-induced arthritis, phosphodiesterase 4, Apremilast, regulatory T and Th17 cells 


\section{INTRODUCTION}

Rheumatoid arthritis (RA) is a chronic inflammatory bonedestructive disorder with autoimmune features (1). It is driven by diverse cellular and humoral immune responses, resulting in articular synovial inflammation and bone destruction $(2,3)$. Type II collagen (CII)-induced arthritis (CIA) is a valuable animal model for investigating the pathological development of RA and for evaluating potential therapies (4). Similar to RA clinical manifestations in humans, the CIA model exhibits joint swelling, and histological examination confirms the presence of synovitis, periosteal new bone formation, articular bone erosion, and osteopenia (5). The evaluation of potential therapies requires techniques to quantify the severity of disease and pathophysiological response in experimental animals (6-8). Joint inflammation can be visually observed.

Apremilast, an oral phosphodiesterase 4 inhibitor, has been shown to regulate inflammatory mediators. Phosphodiesterase 4 , the dominant phosphodiesterase expressed in immune cells, degrades cyclic AMP (cAMP) into AMP. Phosphodiesterase 4 inhibition, thereby elevates intracellular cAMP, which can downregulate the inflammatory responses through mechanisms such as partially inhibiting expression of inflammatory cytokines and increasing expression of anti-inflammatory mediators such as interleukin-10 (9).

Anti-TNF- $\alpha$ directed biological disease-modifying antirheumatic drugs currently in use (adalimumab, certolizumab pegol, etanercept, golimumab, and infliximab) are highly effective in reducing inflammation and limiting joint destruction (10). However, these treatments are insufficient and not curable (11). Side effects, such as risk for reactivation tuberculosis and malignancy, also limit their use $(11,12)$. Moreover, these treatments are administered via repeated injections with injection site reactions; hence, there is an urgent need for convenient, orally available and safe treatments that are able to reduce the production of TNF- $\alpha$ and other inflammatory mediators. Apremilast, an orally administered PDE4 inhibitor, has been approved in the United State and Europe since 2014 for adult patients with active psoriatic arthritis or plaque psoriasis. Apremilast mainly affects the innate immune system and also decreases the production of Th1, Th2, and Th17 cytokines, whereas its role in B cells and IL- 6 production is minor. Given $\mathrm{T}$ effector cells (Th1 and Th17) predominately affect the pathogenesis and development of autoimmune arthritis $(13,14)$, we aimed to determine whether Apremilast, a PDE4 inhibitor, can prevent and even treat autoimmune arthritis through suppressing $\mathrm{T}$ effector cells and/or modulating regulatory $\mathrm{T}$ (Treg) cells.

\section{MATERIALS AND METHODS}

\section{Mice}

Male DBA/1J mice were purchased from The Jackson Laboratory (Bar Harbor, ME, USA). C57BL/6 Foxp3 ${ }^{\text {gfp }}$ reporter mice were generously provided by Dr. Talil Chatilla (University of California, Los Angeles). DBA/1J-FoxP3 ${ }^{\text {ffp }}$ mice were produced by backcrossing C57BL/6 Foxp3 $3^{\text {frp }}$ mice with DBA/1J mice for 13 generations. Severe combined immunodeficiency (SCID) mice were purchased from Model Animal Research Center of Nanjing
University. All animals were treated according to the National Institutes of Health guidelines for the use of experimental animal with the approval of Penn State University Hershey Medical Center and the first affiliated hospital at Zhejiang University for the Use and Care of Animals. Our experiment was followed by the standard biosecurity and institutional safety procedures in both institutes (SUZ15-01-2).

\section{Induction of Arthritis}

Bovine CII (Condrex Inc., WA, USA) was emulsified with an equal volume of complete Freund's adjuvant (Sigma, MO, USA) containing $4 \mathrm{mg} / \mathrm{ml}$ of heat-denatured Mycobacterium (BD Biosciences, CA, USA). DBA1/J and or DBA/1J-Foxp3 $3^{\text {frp }}$ mice were immunized via an intradermal injection at the base of the tail with $50 \mu \mathrm{l}$ of emulsion (CII $100 \mu \mathrm{g} /$ mouse). The mice were then euthanized by $\mathrm{CO}_{2}$ asphyxiation and cervical dislocation at indicated times. To determine whether Apremilast can prevent and even treat CIA, mice received Apremilast (Celgene Corporation, NJ, USA) orally daily for the continuous 10 days. Apremilast ( 5 or $25 \mathrm{mg} / \mathrm{kg}$ ) was given to DBA/1J around 14 days after CII immunization. As a negative control, the medium alone without Apremilast (0.5\% carboxymethyl cellulose, $0.25 \%$ Tween 80 ) was administered by oral gavage.

\section{Evaluation of Clinical Arthritis}

Clinical signs of arthritis were evaluated every 2-3 days after immunization to determine arthritis incidence. Each paw was evaluated and scored individually for severity of arthritis using a previously described scoring system (scale $0-4)(4,15)$. The scores for each paw were summed to yield a total arthritis severity score per mouse, with a maximum score of 16 for each animal. Each paw score was judged as follows: 0 , no signs of arthritis, 1 , mild swelling confined to the tarsal bones or ankle joint, 2 , mild swelling extending from the ankle to the tarsal bones, 3 , moderate swelling extending from the ankle to the metatarsal joints, and 4 , severe swelling encompassing the ankle, foot, and digits, or ankylosis of the limb.

\section{Histopathologic Evaluation of the Joints}

After the mice were sacrificed on day 56, the hind limbs were collected. Following routine fixation, decalcification, and paraffin embedding, tissue sections were prepared and stained with hematoxylin and eosin. All slides were evaluated by blinded investigators with regard to the experimental conditions. The extent of synovitis, pannus formation, and bone/cartilage destruction was determined using a graded scale, as follows (15): grade 0 , no signs of inflammation; 1 , mild inflammation with hyperplasia of the synovial lining without cartilage destruction; $2-4$, increasing degrees of inflammatory cell infiltration and cartilage/bone destruction.

\section{Cell Stimulation, Suppression Assay, and Differentiation In Vitro}

$\mathrm{CD} 4{ }^{+} \mathrm{CD} 25^{+}$cells (Treg) sorted from the spleen in DBA/1J mice were pretreated with $0.1 \mu \mathrm{M}$ Apremilast or DMSO for $24 \mathrm{~h}$, then cultured under a condition polarizing Th17 cells with irradiated 
APC $(1: 1)$, soluble anti-CD3 $(1 \mu \mathrm{g} / \mathrm{ml})$, soluble CD28 $(1 \mu \mathrm{g} / \mathrm{ml})$, and IL-6 $(20 \mathrm{ng} / \mathrm{ml})$ for another 3 days. After that, the cells were harvested, tested for IL-17a and Foxp3-GFP expression by flow cytometry. We also measured CD126 expression on Treg after pretreatment with $0.1 \mu \mathrm{M}$ Apremilast or DMSO by flow cytometry.

To measure in vitro suppression, CFSE-labeled T cells were stimulated with soluble anti-CD3 $(0.25 \mu \mathrm{g} / \mathrm{ml})$ with irradiated APC (1:1). To the responder cells, Treg (pretreated with DMSO) or Treg (pretreated with $0.1 \mu \mathrm{M}$ Apremilast) stimulated with or without IL-6 $(20 \mathrm{ng} / \mathrm{ml})$ was added at a 1:1 ratio, and suppression of cycling CFSE-labeled T cells was assessed flow cytometry.

\section{Flow Cytometry}

Cells were isolated from the spleens and draining lymph nodes of arthritic mice on day 56 after CII immunization. The following fluorescence-conjugated mouse antibodies were used for flow cytometric analysis: from BioLegend (San Diego, CA, USA): mouse PerCP/Cy5.5-anti-CD4 (GK1.5), PE-CD25 (3C7), PE-IFN- $\gamma$ (XMG1.2), AlexaFluor 647-IL-17a (TC11-18H10.1). Cell subset was stained with $\mathrm{mAbs}$ and isotype control indicated above and analyzed on a FACS Calibur flow cytometer using Cell Quest Software (Becton Dickinson). For intracellular staining, including IFN- $\gamma$ and IL-17a, cells were first stained with surface marker CD4, and further fixed and permeabilized for intracellular staining. Plot figures were prepared using FlowJo Software (Tree Star Inc., Ashland, OR, USA).

\section{ELISA for Anti-Mouse CII Antibody}

Serum levels of mouse anti-bovine CII antibody (total IgG, IgG1, IgG2a, and IgG2b, and IgG3) were measured according to a modification of the previously published protocol (16). Briefly, coated 96-well plates were coated with $1 \mu \mathrm{g} / \mathrm{ml}$ bovine CII and incubated at $4^{\circ} \mathrm{C}$ overnight, followed by blocking with a $2 \mathrm{~h}$ incubation at $4^{\circ} \mathrm{C}$ with $3 \% \mathrm{BSA}$ in phosphate-buffered saline (PBS, pH 7.4). Serum samples diluted at 1:1,000 in PBS containing 1\% BSA were incubated $1.5 \mathrm{~h}$ at $37^{\circ} \mathrm{C}$. Goat anti-mouse IgG, IgG1, IgG2a, IgG2b, and IgG3 conjugated to HRP (Southern Biotechnology, Birmingham, AL, USA) diluted at 1:4,000 were incubated for $1.5 \mathrm{~h}$ at $37^{\circ} \mathrm{C}$ to detect anti-CII antibody isotypes and IgG subclasses. A pre-warmed $o$-phenylenediamine buffer was used to visualize the reaction. The optical density at $490 \mathrm{~nm}$ was read with a Molecular Devices plate reader (Molecular Devices, Menlo Park, CA, USA). Antibody levels were expressed as units with reference to a standard serum.

\section{Micro-Computed Tomographic Image Analysis}

Mice were anesthetized with $2 \%$ isoflurane. The high-resolution micro-computed tomography (micro-CT) system (VivaCT 40, Scanco, Switzerland) was used to acquire in vivo imaging of the three-dimensional bone. The scans were performed with $3.6 \mathrm{~mm}$ length including entire single mouse foot with the following parameters: $17.5 \mu \mathrm{m}$ voxel size at $55 \mathrm{kV}, 145 \mu \mathrm{A}, 200 \mathrm{~ms}$ integration time, 211 image slices. The micro-CT images were converted to 8-bit, imported into Mimics software (Materialise, Belgium), then filtered using discrete Gaussian filtering (variance $=1$; $\max$ kernel width $=1$ ). Volumes of interest in the metatarsophalangeal joint were used to quantify bone erosion as follows. Second through fourth metatarsal and phalangeal bones were segmented from surrounding soft tissue using a consistent image intensity threshold. Three volumes of interest were set with $\pm 1 \mathrm{~mm}$ length in the distal and the proximal direction from the center of each metatarsophalangeal joint. These volumes of interest were oriented consistently based on the $3 \mathrm{D}$ longitudinal axis of the third metatarsal. The bone volumes of the three metatarsophalangeal joints were then calculated.

\section{Inflamed Synovial Tissue-Mediated Humanized Animal Model}

Sponge-cartilage complex, containing a piece of cartilage with RA synovial fibroblasts (RASFs, $5 \times 10^{5}$ ) from RA patients was implanted into the flank skin of an SCID mouse (primary implant). Surgery was performed on the condition of isoflurane anesthesia. The skin incision was made with a sterile scalpel. One drop of bupivacaine was applied to the incision before closure for local analgesia. The mice were monitored for signs of discomfort. Two RA patients (one female, 55 years old, with RA for 12 years, another female, 54 years old, with RA for 3 years) were recruited from the first affiliated hospital at Zhejiang University with IRB approval. The written informed consent was obtained from these two patients. We also inserted cartilage without RASFs under the skin of the contralateral flank (contralateral implant). After the humanized synovitis model had implanted, Apremilast (25 mg/kg, once daily) was given to SCID mice on the day when cartilage with RASFs was implanted for the continuous 10 days. We removed the implants after 60 days for evaluation. They were stained by standard hematoxylin and eosin. Invasion scores were classified as a previous report (17).

\section{Statistics}

Data are expressed as mean \pm SEM. Data were analyzed using the unpaired $t$-tests (Mann-Whitney) between two groups or oneway ANOVA for comparison among multiple groups followed by Turkey's test as appropriate. Differences were considered statistically significant when $p<0.05$.

\section{RESULTS}

\section{Apremilast Therapy Delayed Arthritis Onset and Reduced Arthritis Scores in CIA Model}

Apremilast has been shown to regulate inflammatory mediators. We first tested whether Apremilast (5 or $25 \mathrm{mg} / \mathrm{kg}$ ) can change arthritis onset. Both doses of Apremilast significantly delayed arthritis onset and markedly reduced arthritis scores in the CIA model. High dose of Apremilast $(25 \mathrm{mg} / \mathrm{kg})$ was superior to low dose $(5 \mathrm{mg} / \mathrm{kg})$ in reducing arthritis scores (Figures 1A,B). We then examined serum anti-CII antibody and found that the total serum IgG, IgG2a, and IgG2b were decreased in the Apremilast $(5 \mathrm{mg} / \mathrm{kg}$ ) treatment group, compared with the vehicle control group. In a dose response, the 
A

$\rightarrow$ CIA Model

$\rightarrow \mathrm{CIA}+\mathrm{CMC}$

$\rightarrow \mathrm{ClA}+$ Apremilast $5 \mathrm{mg} / \mathrm{kg}$

$\rightarrow \mathrm{ClA}+$ Apremilast $25 \mathrm{mg} / \mathrm{kg}$

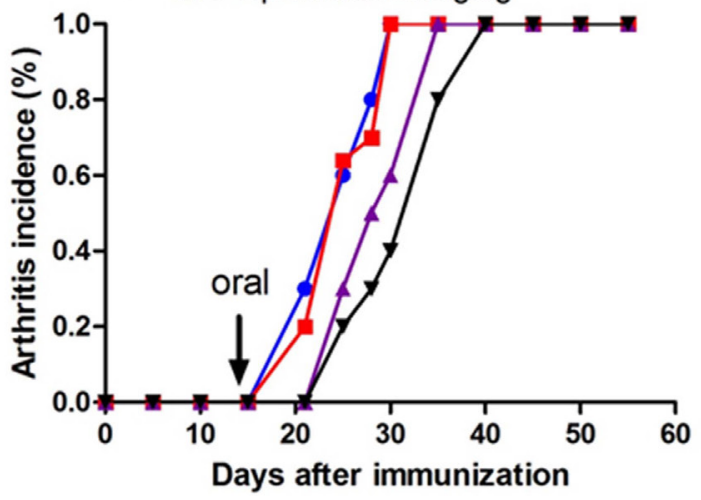

C
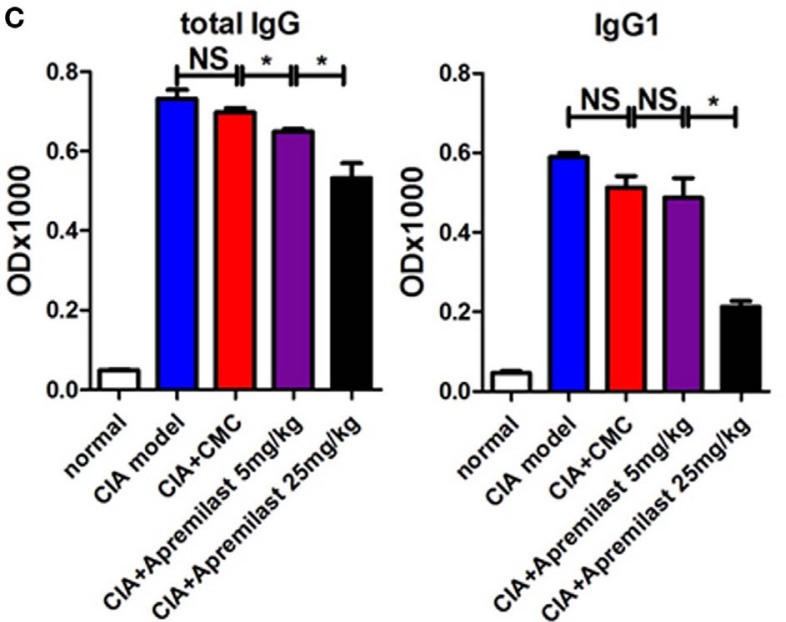
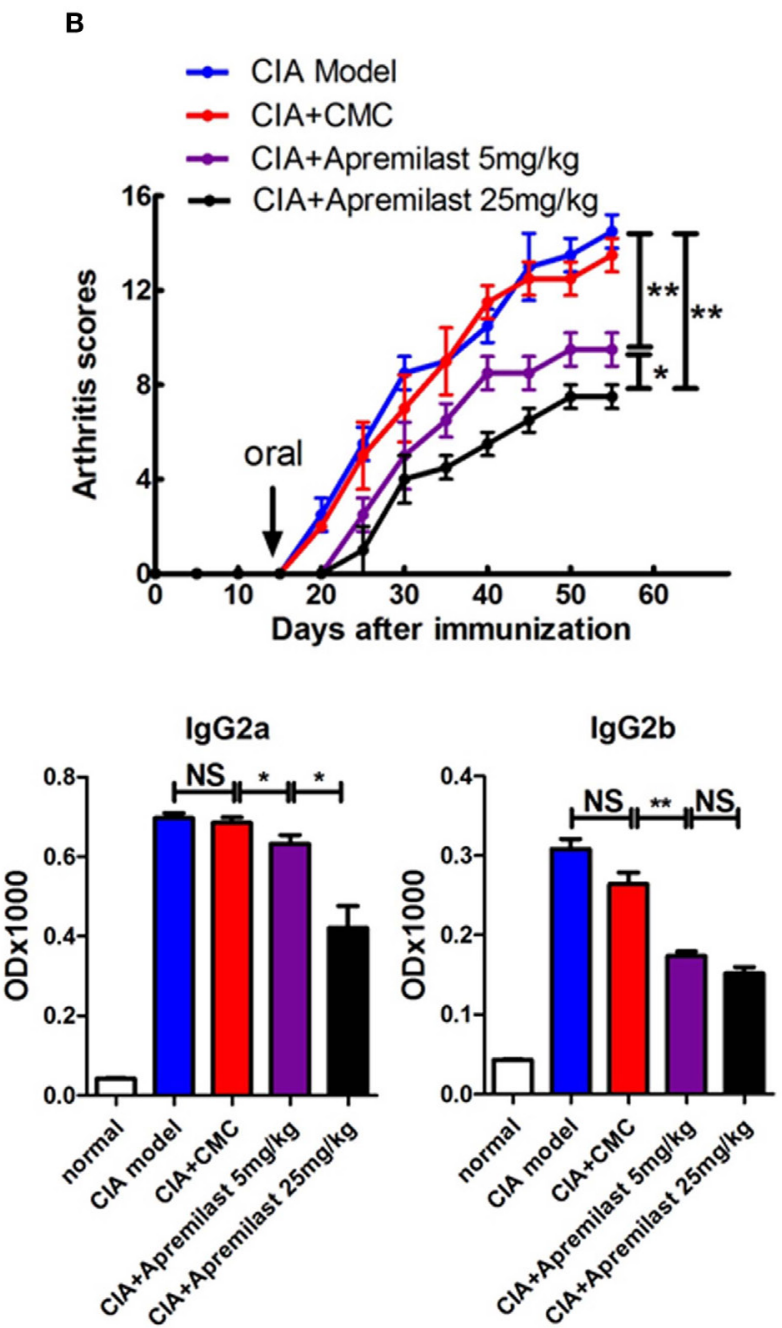

FIGURE 1 | Apremilast delayed arthritis onset and reduced arthritis scores in the collagen-induced arthritis (CIA) model. DBA/1J-FoxP3 gfp mice were immunized with type II collagen emulsified with Freund's complete adjuvant. On day 14 after immunization, Apremilast (5 or $25 \mathrm{mg} / \mathrm{kg}$ ) was given orally once daily for 10 days. Vehicle alone ( $0.5 \%$ carboxymethyl cellulose, $0.25 \%$ Tween $80, \mathrm{CMC})$ was administered by oral gavage as a negative control. (A) The incidence of arthritis and (B) arthritis severity scores were determined at various time points after immunization. (C) Total serum IgG, IgG1, IgG2a, and lgG2b anti-mouse type II collagen antibody levels were measured by enzyme-linked immunosorbent assay. The data indicate the mean \pm SEM of five mice per group from two independent experiments. Data were analyzed using the one-way ANOVA for comparison among multiple groups, followed by Turkey's test $\left({ }^{*} p<0.05,{ }^{* *} p<0.01\right)$.

total serum IgG and IgG2a levels were lower in the Apremilast $(25 \mathrm{mg} / \mathrm{kg}$ ) group compared with the Apremilast $(5 \mathrm{mg} / \mathrm{kg}$ ) group. IgG1 was only decreased in the Apremilast $(25 \mathrm{mg} / \mathrm{kg}$ ) group (Figure 1C), but IgG3 did not change in the Apremilast treatment groups (data not shown).

\section{Apremilast Reduced Arthritis and Prevented Bone Erosion in CIA Model}

To determine whether Apremilast can prevent CIA mice from bone erosion, joint HE staining and micro-CT scan was applied. Histological changes in the whole ankle joints demonstrated a significant decrease in synovitis, pannus formation, and destruction of bone and cartilage after treatment with Apremilast (Figure 2). There was no difference between the lower dose group and the high dose group for histological findings (Figure 2). Quantitative analyses of the whole ankle confirmed a similarly significant increase in the bone volume of the metatarsophalangeal joint in the Apremilast treatment group by micro-CT analysis, compared with vehicle control (Figure 3).

\section{Apremilast Reduced Arthritis Through Suppression of Th1 and Th17 Cells and Enhancement of Treg Cells Differentiation}

As the Th1 and Th17 cytokines are the most important proinflammatory response involved in the development of CIA, we next investigated the impact of Apremilast on these effector cells. The analysis clearly demonstrated that Th17 cells and Th1 cells from draining lymph nodes were both decreased in the low and high dose 

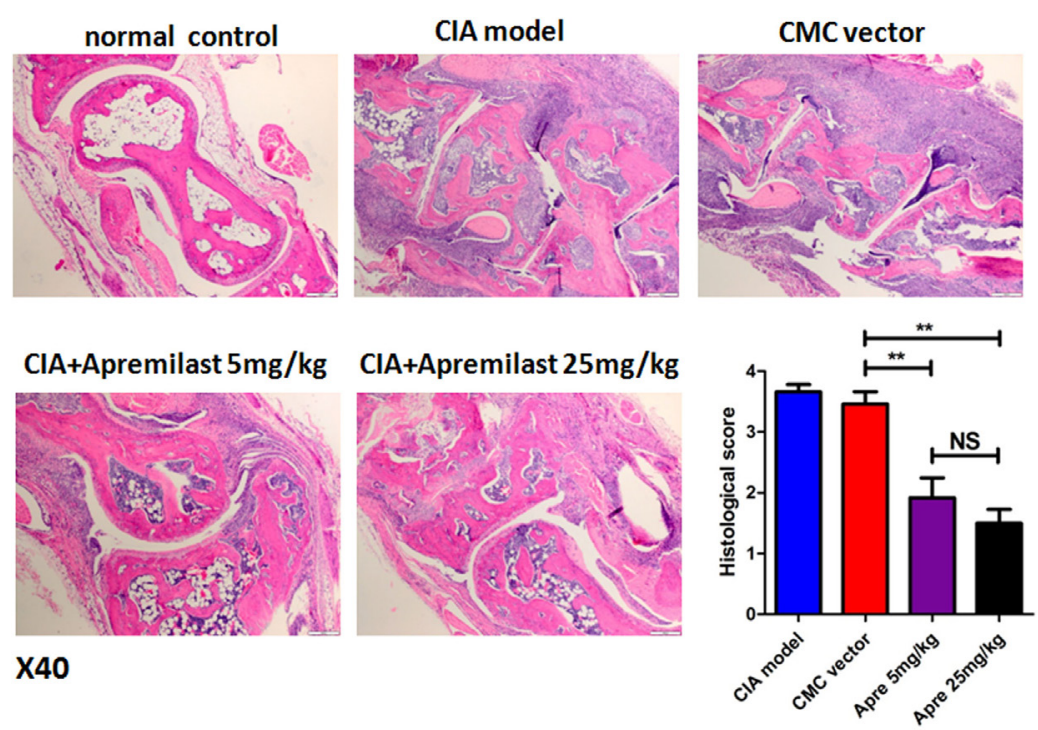

FIGURE 2 | Apremilast administration reduced arthritis pathology in collagen-induced arthritis (CIA) model. Ankle joint sections were stained with hematoxylin and eosin 56 days after the primary immunization and evaluated for the histopathologic features of synovitis, pannus, and erosion (representative results were shown; 40x) in Apremilast (5 or 25 mg/kg), CMC vehicle alone, CIA model, and normal groups. Results were expressed quantitatively as the histopathology score (right lower panel). The data indicate the mean \pm SEM of two separated experiments, each group with five mice. Data were analyzed using the one-way ANOVA for comparison among multiple groups, followed by Turkey's test $\left({ }^{\star \star} p<0.01\right)$.

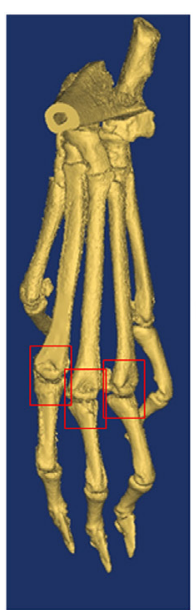

CMC vector

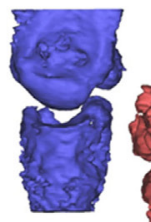

\section{Normal}

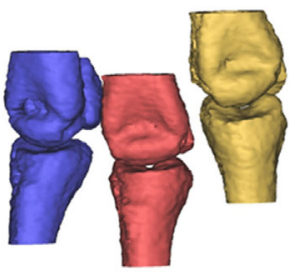

CIA Model
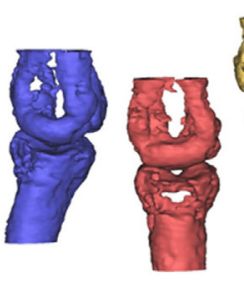
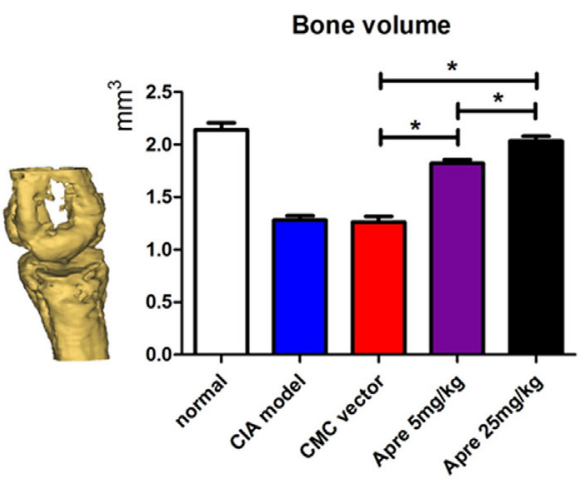

Apremilast $5 \mathrm{mg} / \mathrm{kg}$

Apremilast 25mg/kg
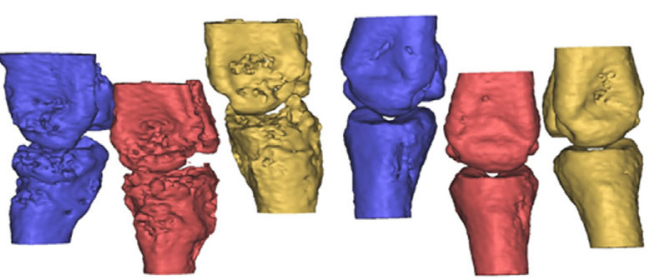

FIGURE 3 | Apremilast prevented bone erosion in collagen-induced arthritis (CIA) model. Apremilast ( 5 or $25 \mathrm{mg} / \mathrm{kg}$ ), CMC vehicle alone, CIA model, and normal groups mice were killed on day 56, the right hind limbs were collected and analyzed by the high-resolution micro-computed tomography (micro-CT) system (VivaCT 40). Second through fourth metatarsal and phalangeal bones were segmented from surrounding soft tissue using a consistent image intensity threshold. Three volumes of interest were set with $\pm 1 \mathrm{~mm}$ length in the distal and proximal direction from the center of each metatarsophalangeal joint. The bone volumes of the three metatarsophalangeal joints were then calculated. Representative micro-CT images were shown. The summary data are shown in the left panel. The data indicate the mean \pm SEM of five mice per group from two independent experiments $\left({ }^{*} p<0.05\right)$. Data were analyzed using the one-way ANOVA for comparison among multiple groups, followed by Turkey's test. 


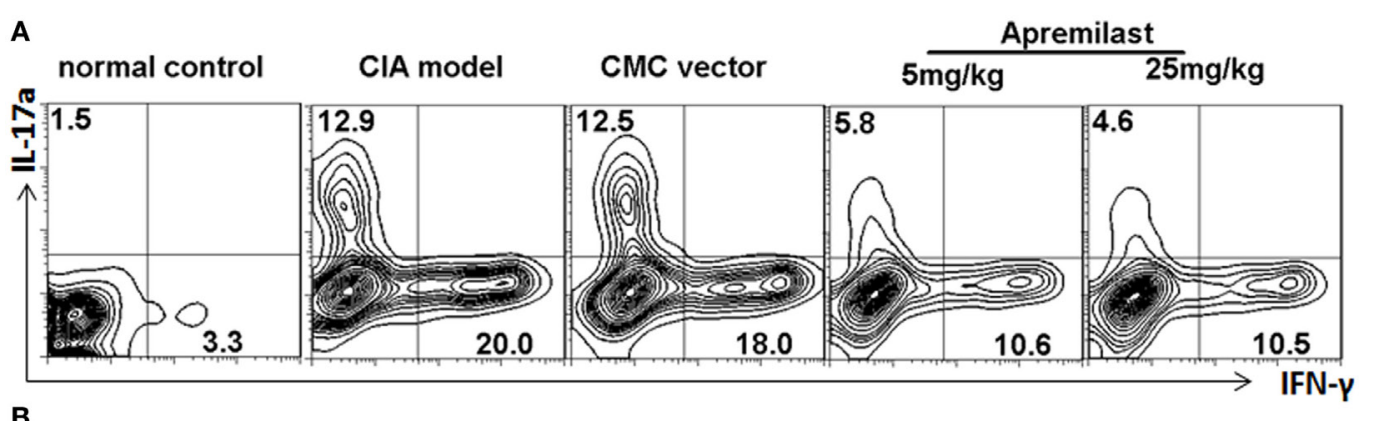

B

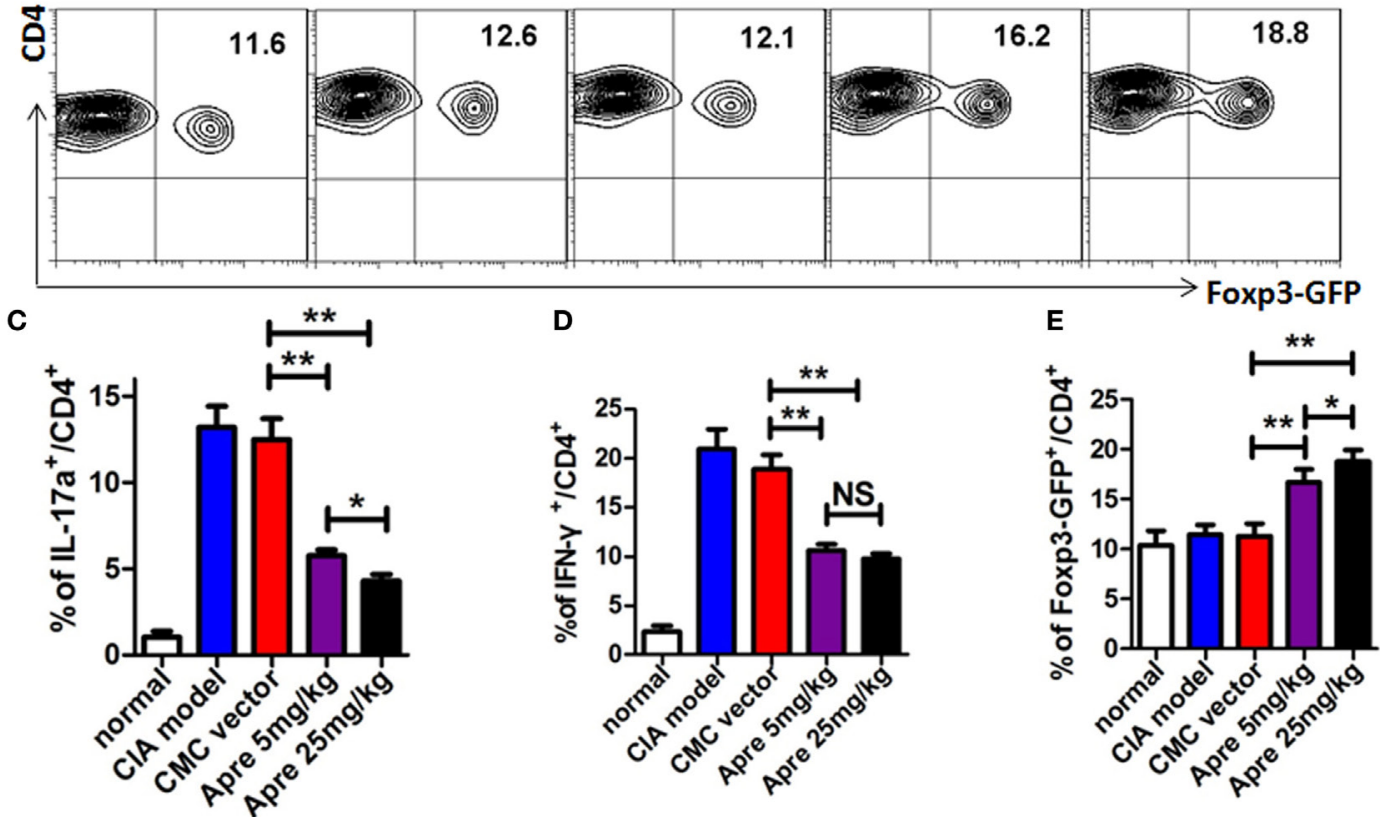

FIGURE 4 | Apremilast reduced arthritis through suppression of Th1 and Th17 cells and enhancement of regulatory T cell differentiation. Cells were isolated from the draining lymph nodes of arthritic mice on day 56 after type II collagen immunization in Apremilast (5 or 25 mg/kg), CMC vehicle alone, collageninduced arthritis (CIA) model, and normal groups. The expression of IL-17a, IFN- $\gamma$ (A), and Foxp3 ${ }^{+}-$GFP (B) on CD4+ T cells were measured in draining lymph node cells by flow cytometry. The summary data are shown in the lower panel (C-E). The data indicate the mean \pm SEM of five mice per group from two independent experiments $\left({ }^{*} p<0.05,{ }^{* *} p<0.01\right)$. Data were analyzed using the one-way ANOVA for comparison among multiple groups, followed by Turkey's test.

Apremilast groups (Figures 4A,C,D) (Figure S2 in Supplementary Material). No difference between two groups was observed. Foxp ${ }^{+}$ Treg cells play a crucial role in the maintenance of immune tolerance and prevention of RA. We therefore tested whether Foxp3 ${ }^{+}$ Treg cells might also by affected by the Apremilast treatment in CIA. We noted that Treg cells were enhanced in both the low and high dose Apremilast groups (Figures 4B,E). High dose Apremilast treatment resulted in a higher enhancement of Treg cells in CIA (Figures 4B,E) (Figure S2 in Supplementary Material).

\section{Apremilast Promoted Treg by Maintaining the Foxp3 Stability and Prevented Treg Conversion to Th17 Cells In Vitro}

We also determined the mechanisms thereby Apremilast promoting Treg cells. We isolated the natural Treg cells from spleen of
DBA1 mice, pretreated with Apremilast or DMSO $24 \mathrm{~h}$, then cultured under a condition polarizing Th17 cells for 3 days in vitro. We demonstrated that Treg pretreated with Apremilast had a higher Foxp3 expression and lower IL-17a expression when compared with DMSO group under the simulation of IL-6 (Figure 5A). As we known, IL- 6 can induce natural Treg to become Th17 cells through IL-6R (CD126) mediated signaling $(8,13,18)$. We found that the CD126 expression was quite lower in Treg pretreated with Apremilast than that pretreated with DMSO control (Figure 5B). Furthermore, Treg pretreated with Apremilast or DMSO had a similar function in suppressing the $\mathrm{T}$ cell proliferation without IL- 6 treatment. However, the ability of Treg to suppress the T cell proliferation was significantly decreased after stimulation with IL-6, while Treg pretreated with Apremilast mostly sustained the suppressive activity in the condition of pro-inflammatory IL-6 (Figure 5C). 

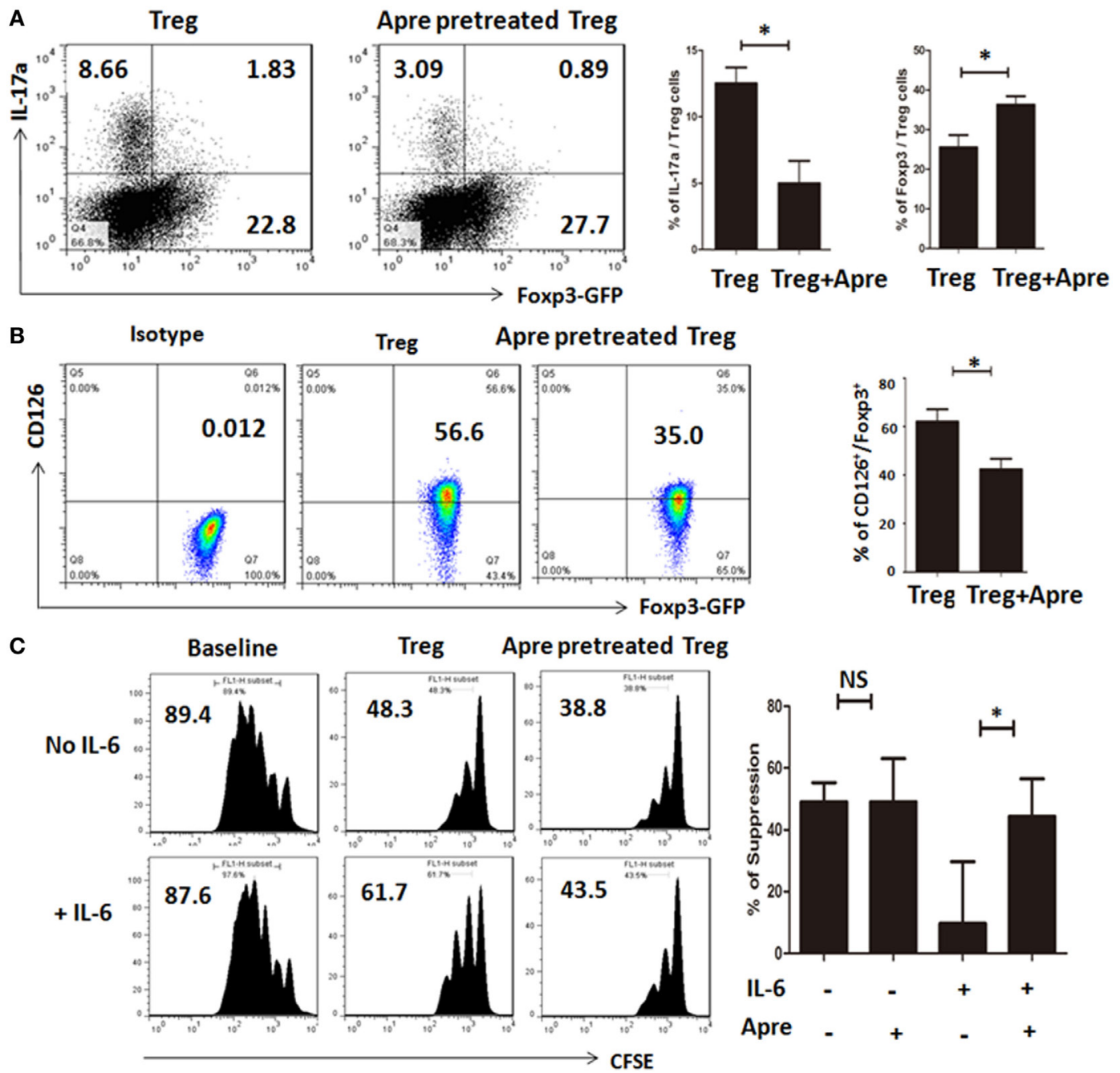

FIGURE 5 | Apremilast promoted Treg by maintaining the Foxp3 stability and prevented Treg conversion to Th17 cells in vitro. (A) CD4+CD25+ cells (Treg) were isolated with cell sorting and pretreated with $0.1 \mu \mathrm{M}$ Apremilast or DMSO for $24 \mathrm{~h}$, cultured under a condition polarizing Th17 cells for another 3 days. Then, the cells were harvested, tested for IL-17a and Foxp3-GFP expression by flow cytometry (mean \pm SEM of three independent experiments, Mann-Whitney). (B) The CD126 expression was measured on Treg after pretreatment with $0.1 \mu \mathrm{M}$ Apremilast or DMSO by flow cytometry and qPCR (mean \pm SEM of three independent experiments, Mann-Whitney). (C) CFSE-labeled T cells were cultured with Treg (pretreated with DMSO) or Treg (pretreated with $0.1 \mu \mathrm{M}$ Apremilast) in the presence of IL-6 or not at a 1:1 ratio, and suppression of cycling CFSE-labeled T cells was assessed flow cytometry (mean \pm SEM of three independent experiments,

Mann-Whitney) $\left({ }^{*} p<0.05\right)$.

\section{Apremilast Treatment Inhibited RASFs from Migrating and Destroying Cartilage in the Humanized Animal Model}

To determine its clinical relevance, we also developed a humanized animal model to test whether Apremilast can modulate human inflamed synovial tissue-mediated disease in vivo. We implanted a sponge-cartilage complex, containing cartilage and RASFs to determine whether Apremilast can suppress the human RASFs function in vivo. Cells were phenotyped by flow cytometry to confirm bona fide RASFs (Figure S1 in Supplementary Material). After the humanized synovitis model was established, Apremilast ( $25 \mathrm{mg} / \mathrm{kg}$, once daily) was given to SCID mice for the continuous 10 days. The cartilage damage and scores were evaluated as previously described in Ref. (17). We demonstrated that Apremilast administration significantly suppressed cartilage destruction and RASFs migration (Figure 6), processes that both are relevant to RA.

\section{DISCUSSION}

In this study, our results demonstrated that oral Apremilast therapy delayed arthritis onset and reduced arthritis scores in the CIA model with a dose-dependent effect. Crucial pathogenic antibodies of anti-CII IgG, IgG1, IgG2a, and IgG2b were all reduced after Apremilast administration. Notably, Apremilast markedly prevented bone erosions in CIA mice. These results 

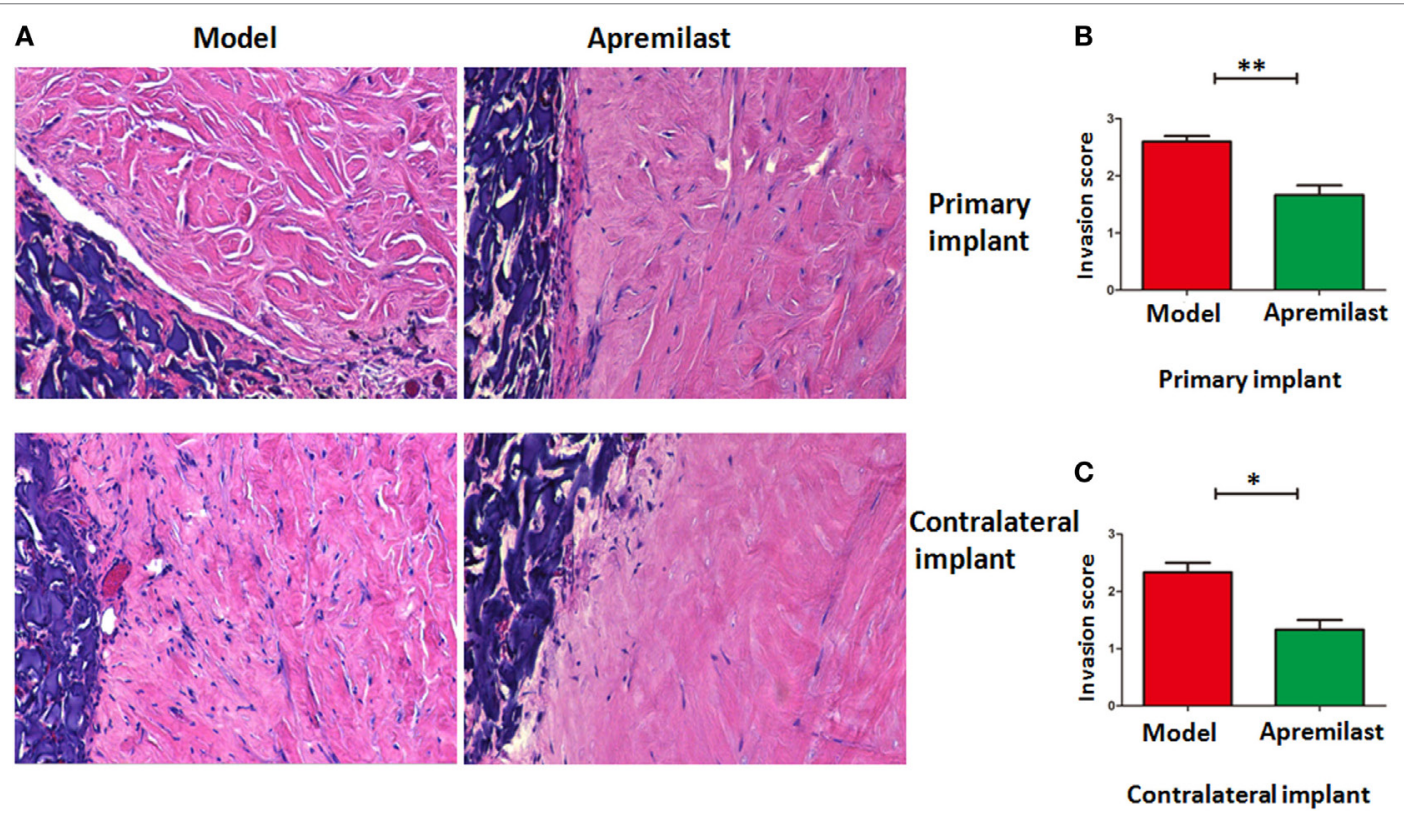

FIGURE 6 | Apremilast treatment inhibited rheumatoid arthritis (RA) synovial fibroblast (RASFs) from migrating and destroying cartilage in the humanized animal model. Sponge-cartilage complex, containing cartilage with synovial fibroblasts from RA patients (RASFs), was implanted into the flank skin of a severe combined immunodeficiency (SCID) mouse (primary implant). We also inserted cartilage without RASFs under the skin of the contralateral flank (contralateral implant). After the humanized synovitis model was established, Apremilast $(25 \mathrm{mg} / \mathrm{kg}$, oral, once daily) was given to SCID mice for 10 continuous days. We removed the implants for evaluation after 60 days. Invasion scores are classified as the previous report. (A) Apremilast treatment can inhibit the ability of RASFs from migrating to and destroying cartilage. $(\mathbf{B}, \mathbf{C})$ Compared with the model group $(n=6)$, Apremilast treatment $(n=6)$ significantly reduced the invasion scores in both primary implant and contralateral implant. The data indicate the mean \pm SEM from two independent experiments $\left({ }^{*} p<0.05\right.$, $\left.{ }^{* *} p<0.01\right)$. Data were analyzed using unpaired $t$-tests (Mann-Whitney).

imply that orally administered Apremilast should be evaluated as a treatment option in patients with RA.

A previous report has shown that intraperitoneal injection of Apremilast can ameliorate arthritis (19). However, this study did not investigate whether Apremilast can prevent bone loss in CIA. Given oral administration is clinically feasible when translating the work to human studies, our study using oral administration adds evidence for the future study of Apremilast treatment in patients with RA.

Given T effector cells (Th1 and Th17) predominately affect the pathogenesis and development of autoimmune arthritis $(15,20)$, we investigated the hypothesis that Apremilast suppressed Th17 cells and Th1 cells. As expected, Apremilast significantly decreased the frequency of these pathogenic T effector cells. Conversely, Apremilast upregulated the frequencies of Foxp $3^{+} \mathrm{CD}^{+}$Treg cells. The Treg/Teff cell balance is crucial for the development and progression of autoimmune arthritis and other autoimmune diseases $(6,21,22)$. Furthermore, we also demonstrated that Apremilast could promote Treg cells by maintaining the Foxp3 stability and preventing Treg cells conversion to Th17 cells in vitro. Moreover, Apremilast affects IL-6/IL-6R signaling on Treg cells.

Our study has demonstrated clear effectiveness and a doseresponse of Apremilast in the CIA model. While Apremilast has been approved to treat patients with psoriatic arthritis, its therapeutic effect on patients with RA has also been explored in a double-blinded, placebo-controlled phase 2 clinical trial in patients with active RA who were inadequate responders to methotrexate (MTX). Apremilast efficacy was not observed in these patients. The trial did show that Apremilast was well tolerated in patients with longstanding active RA with mean disease duration of greater than 8 years (23). Reasons for this discrepancy between mouse models and patients with RA could be explained with some reasons. Apremilast targets predominantly the Th1 and Th17 response that is important in early RA. However, after the disease is established with a humoral response and the synovial pannus formation, Apremilast may not be as effective. Another possibility for the discrepancy is that the dose used in the clinical trials was too low as a previous study has demonstrated that Apremilast can reduce TNF- $\alpha$ production by human synovial cells from RA patients undergoing joint replacement surgery (19). Additional explanation for the lack of efficacy of Apremilast in RA patients who are MTX inadequate responders is that there is a partial overlap of the mechanisms of action of MTX and Apremilast (24). The PDE4D immunostaining within RA synovia was not elevated in the subgroup of patients with poor response to MTX therapy (25). It is possible that Apremilast may be more appropriate for a different RA population. Further clinical trials are needed to investigate the efficacy of Apremilast in patients with RA with a focus on patients with the early newly diagnosed disease. 
Taken together, our study demonstrates that oral administration of Apremilast can ameliorate nonclinical arthritis and can protect against bone damage. We also found that Apremilast regulates the balance between Treg cells and Teff cells in the CIA. These observations may inform the design of clinical trials of Apremilast in patients with early RA, for example prior to MTX exposure, when Th1, Th17, and Treg modulation via PDE4 inhibition may have a greater impact.

\section{ETHICS STATEMENT}

All animals were treated according to the National Institutes of Health guidelines for the use of experimental animal with the approval of PSU Hershey and the first affiliated hospital at Zhejiang University for the Use and Care of Animals. Patients with RA were recruited with consent from the first affiliated hospital at Zhejiang University with IRB approval.

\section{AUTHOR CONTRIBUTIONS}

SZ: conception and design of the study; final approval of manuscript; WC, JW, ZX, WQ, JM, FH, HW, GL, and JL: performed experiments; WC, ZX, and SZ: data analysis and interpretation; WC and SZ: wrote the manuscript; RJ and PS: critically revised the manuscript.

\section{REFERENCES}

1. Liu Y, Pan YF, Xue YQ, Fang LK, Guo XH, Guo X, et al. uPAR promotes tumor-like biologic behaviors of fibroblast-like synoviocytes through PI3K/ Akt signaling pathway in patients with rheumatoid arthritis. Cell $\mathrm{Mol}$ Immunol (2017) 15(2):171-81. doi:10.1038/cmi.2016.60

2. Peng WX, Zhu SL, Zhang BY, Shi YM, Feng XX, Liu F, et al. Smoothened regulates migration of fibroblast-like synoviocytes in rheumatoid arthritis via activation of Rho GTPase signaling. Front Immunol (2017) 8:159. doi:10.3389/ fimmu.2017.00159

3. Zhu SL, Huang JL, Peng WX, Wu DC, Luo MQ, Li QX, et al. Inhibition of smoothened decreases proliferation of synoviocytes in rheumatoid arthritis. Cell Mol Immunol (2017) 14(2):214-22. doi:10.1038/cmi.2015.67

4. Brand DD, Latham KA, Rosloniec EF. Collagen-induced arthritis. Nat Protoc (2007) 2(5):1269-75. doi:10.1038/nprot.2007.173

5. Barck KH, Lee WP, Diehl LJ, Ross J, Gribling P, Zhang Y, et al. Quantification of cortical bone loss and repair for therapeutic evaluation in collagen-induced arthritis, by micro-computed tomography and automated image analysis. Arthritis Rheum (2004) 50(10):3377-86. doi:10.1002/art.20557

6. Kong N, Lan Q, Chen M, Wang J, Shi W, Horwitz DA, et al. Antigen-specific transforming growth factor $\beta$-induced Treg cells, but not natural Treg cells, ameliorate autoimmune arthritis in mice by shifting the Th17/Treg cell balance from Th17 predominance to Treg cell predominance. Arthritis Rheum (2012) 64(8):2548-58. doi:10.1002/art.34513

7. Kong N, Lan Q, Chen M, Zheng T, Su W, Wang J, et al. Induced T regulatory cells suppress osteoclastogenesis and bone erosion in collagen-induced arthritis better than natural T regulatory cells. Ann Rheum Dis (2012) 71(9):1567-72. doi:10.1136/annrheumdis-2011-201052

8. Zhou X, Kong N, Wang J, Fan H, Zou H, Horwitz D, et al. Cutting edge: all-trans retinoic acid sustains the stability and function of natural regulatory $\mathrm{T}$ cells in an inflammatory milieu. J Immunol (2010) 185(5):2675-9. doi:10.4049/jimmunol.1000598

9. Schafer P. Apremilast mechanism of action and application to psoriasis and psoriatic arthritis. Biochem Pharmacol (2012) 83(12):1583-90. doi:10.1016/j. bcp.2012.01.001

\section{FUNDING}

This work is supported in part by the grants from the Celgene Foundation, National Natural Science Foundation of China (81701600), and Zhejiang Provincial Natural Science Foundation of China (LQ17H100001).

\section{SUPPLEMENTARY MATERIAL}

The Supplementary Material for this article can be found online at https://www.frontiersin.org/articles/10.3389/fimmu.2018.01662/ full\#supplementary-material.

FIGURE S1 | Phenotypic characteristic of and rheumatoid arthritis (RA) synovial fibroblasts (RASFs) gained from patients with active RA. RASFs were isolated from digested synovial tissue from two patients with RA. The following fluorescence-conjugated mouse anti-human antibodies were used: PE-antiCD55 and AlexaFluor 647-anti-CD90. Cell subset was stained with mAbs indicated above and analyzed on a FACS Calibur flow cytometer.

FIGURE S2 | The absolute number of Th17, Th1, and Treg cells in the draining lymph node. The collagen-induced arthritis treated with CMC (vector control, $n=5$ ), Apremilast (5 mg/kg, $n=5$ ), or Apremilast (25 mg/kg, $n=5)$. The lymphocytes were harvested from the draining lymph node on day 56 after $\mathrm{Cll}$ immunization. The absolute number was calculated by multiplying whole lymphocytes number by CD4 ${ }^{+} \mathrm{T}$ cells percentage in whole lymphocytes and the percentage of Th17, Th1, and Treg cells in CD4+ T cells. Data were analyzed using the one-way ANOVA for comparison among multiple groups, followed by Turkey's test $\left({ }^{*} p<0.05,{ }^{* *} p<0.01\right)$.

10. Taylor PC, Feldmann M. Anti-TNF biologic agents: still the therapy of choice for rheumatoid arthritis. Nat Rev Rheumatol (2009) 5(10):578-82. doi:10.1038/nrrheum.2009.181

11. Smolen JS, Landewe R, Breedveld FC, Buch M, Burmester G, Dougados M, et al. EULAR recommendations for the management of rheumatoid arthritis with synthetic and biological disease-modifying antirheumatic drugs: 2013 update. Ann Rheum Dis (2014) 73(3):492-509. doi:10.1136/annrheumdis2013-204573

12. Burmester GR, Landewe R, Genovese MC, Friedman AW, Pfeifer ND, Varothai NA, et al. Adalimumab long-term safety: infections, vaccination response and pregnancy outcomes in patients with rheumatoid arthritis. Ann Rheum Dis (2017) 76(2):414-7. doi:10.1136/annrheumdis-2016-209322

13. Zheng SG, Wang J, Horwitz DA. Cutting edge: Foxp3+CD4+CD25+ regulatory T cells induced by IL-2 and TGF-beta are resistant to Th17 conversion by IL-6. J Immunol (2008) 180(11):7112-6. doi:10.4049/jimmunol.180.11.7112

14. Zheng SG. Regulatory T cells vs Th17: differentiation of Th17 versus Treg, are the mutually exclusive? Am J Clin Exp Immunol (2013) 2(1):94-106.

15. Chen M, Su W, Lin X, Guo Z, Wang J, Zhang Q, et al. Adoptive transfer of human gingiva-derived mesenchymal stem cells ameliorates collagen-induced arthritis via suppression of Th1 and Th17 cells and enhancement of regulatory T cell differentiation. Arthritis Rheum (2013) 65(5):1181-93. doi:10.1002/art.37894

16. Chu CQ, Song Z, Mayton L, Wu B, Wooley PH. IFNgamma deficient C57BL/6 (H-2b) mice develop collagen induced arthritis with predominant usage of T cell receptor Vbeta6 and Vbeta8 in arthritic joints. Ann Rheum Dis (2003) 62(10):983-90. doi:10.1136/ard.62.10.983

17. Lefevre S, Knedla A, Tennie C, Kampmann A, Wunrau C, Dinser R, et al. Synovial fibroblasts spread rheumatoid arthritis to unaffected joints. Nat Med (2009) 15(12):1414-20. doi:10.1038/nm.2050

18. Lu L, Lan Q, Li Z, Zhou X, Gu J, Li Q, et al. Critical role of all-trans retinoic acid in stabilizing human natural regulatory $\mathrm{T}$ cells under inflammatory conditions. Proc Natl Acad Sci U S A (2014) 111(33):E3432-40. doi:10.1073/ pnas. 1408780111

19. McCann FE, Palfreeman AC, Andrews M, Perocheau DP, Inglis JJ, Schafer P, et al. Apremilast, a novel PDE4 inhibitor, inhibits spontaneous production of tumour necrosis factor-alpha from human rheumatoid synovial cells and 
ameliorates experimental arthritis. Arthritis Res Ther (2010) 12(3):R107. doi:10.1186/ar3041

20. Mellado M, Martinez-Munoz L, Cascio G, Lucas P, Pablos JL, RodriguezFrade JM. T cell migration in rheumatoid arthritis. Front Immunol (2015) 6:384. doi:10.3389/fimmu.2015.00384

21. Chen W, Xu Z, Zheng Y, Wang J, Qian W, Olsen N, et al. A protocol to develop $\mathrm{T}$ helper and Treg cells in vivo. Cell Mol Immunol (2017) 14(12):1013-6. doi:10.1038/cmi.2017.116

22. Nie J, Li YY, Zheng SG, Tsun A, Li B. FOXP3(+) Treg cells and gender bias in autoimmune diseases. Front Immunol (2015) 6:493. doi:10.3389/fimmu. 2015.00493

23. Genovese MC, Jarosova K, Cieslak D, Alper J, Kivitz A, Hough DR, et al. Apremilast in patients with active rheumatoid arthritis: a phase II, multicenter, randomized, double-blind, placebo-controlled, parallel-group study. Arthritis Rheumatol (2015) 67(7):1703-10. doi:10.1002/art.39120

24. Perez-Aso M, Montesinos MC, Mediero A, Wilder T, Schafer PH, Cronstein B. Apremilast, a novel phosphodiesterase 4 (PDE4) inhibitor, regulates inflammation through multiple cAMP downstream effectors. Arthritis Res Ther (2015) 17(1):249. doi:10.1186/s13075-015-0771-6
25. De Groof A, Ducreux J, Humby F, Nzeusseu Toukap A, Badot V, Pitzalis C, et al. Higher expression of TNF $\alpha$-induced genes in the synovium of patients with early rheumatoid arthritis correlates with disease activity, and predicts absence of response to first line therapy. Arthritis Res Ther (2016) 18(1):19. doi:10.1186/s13075-016-0919-z

Conflict of Interest Statement: The authors declare that the research was conducted in the absence of any commercial or financial relationships that could be construed as a potential conflict of interest.

Copyright $\odot 2018$ Chen, Wang, Xu, Huang, Qian, Ma, Wee, Lewis, June, Schafer, Lin and Zheng. This is an open-access article distributed under the terms of the Creative Commons Attribution License (CC BY). The use, distribution or reproduction in other forums is permitted, provided the original author(s) and the copyright owner(s) are credited and that the original publication in this journal is cited, in accordance with accepted academic practice. No use, distribution or reproduction is permitted which does not comply with these terms. 